\section{perifèria}

Número 18(2), diciembre 2013

http://revistes.uab.cat/periferia

\title{
Nomadismo Virtual: analizando sociedades cazadoras-recolectoras a través de Modelos de Simulación Computacional.
}

\author{
Ma Florencia del Castillo Bernal - Universitat Autónoma de Barcelona ${ }^{1}$
}

\section{Resumen}

La Simulación Computacional y los Modelos Basados en Agentes constituyen un nuevo método de investigación para abordar fenómenos sociales complejos. Es desde esta perspectiva que intentamos comprender las dinámicas de movilidad de grupos cazadores recolectores de la Patagonia, basado en la formalización de datos etnoarqueológicos y de diferentes teorías de formación de cazadores-recolectores. Es sobre esta plataforma de formalización sobre la que se desarrollarán los experimentos virtuales.

\begin{abstract}
Computer Simulation and Agent Based Models are a new research method to address complex social phenomena. It is from this perspective that we try to understand the dynamics of mobility of hunter-gatherer groups in Patagonia, based on ethnoarchaeological data formalization and training of different theories of hunter-gatherers. It is over this formalization platform where we will develop the virtual experimentations.
\end{abstract}

Una de las características del análisis arqueológico es explicar por qué el registro arqueológico presenta agrupaciones o patrones, no sólo porque interese en sí mismo el patrón generado, sino también porque que interesa y es significativo para la arqueología es el comportamiento humano que lo ha generado. Es en relación a la variabilidad encontrada en el registro y la variabilidad en su distribución espacial, que la arqueología desde sus comienzos ha intentado explicar las regularidades en la distribución de estas manifestaciones materiales del comportamiento.

En el caso de sociedades nómadas, la identificación de las diferentes formas y niveles de movilidad y en consecuencia, su expresión material, constituye uno de los principales ejes de investigación arqueológica. Es por esto que a partir de 


\section{perifèria}

Número 18(2), diciembre 2013

http://revistes.uab.cat/periferia

estudios etnográficos y etnoarqueológicos, se ha intentado establecer diferentes patrones causales que nos permitan comprender e identificar las variables que actúan en los diferentes patrones y estrategias de movilidad cazadora-recolectora. Los estudios interculturales entre grupos nómadas también nos han permitido obtener datos que habilitan la cuantificación de múltiples variables de movilidad como la distancia, el tiempo, la frecuencia de los asentamientos, la distribución de los asentamientos en relación a la distribución de los recursos o la acumulación de los residuos, entre otras. La mayoría de los modelos teóricos sobre movilidad han sido construidos sobre la base de un amplio rango de variabilidad expresado en los modos de vida de los cazadores-recolectores, por lo tanto, un modelo general y simplificado sobre movilidad nómada puede ser utilizado como estructura de referencia sobre los patrones de movilidad prehistóricos.

Es en base a esta información que nuestro interés se centra en analizar la dinámica de las interacciones sociales desde un nuevo enfoque metodológico que es la Simulación Basada en Agentes. Cuando utilizamos el concepto simulación nos referimos a cómo el comportamiento de un sistema real puede ser reproducido por otro artificial, teniendo en cuenta que estamos construyendo una plataforma sobre la que se experimentará y controlarán las variables del sistema artificial construido, permitiendo así la emergencia de aspectos desconocidos del sistema artificial. La simulación es útil particularmente cuando se analizan sistemas complejos en los que no podemos alcanzar a entender cómo las distintas partes de un sistema de interacción se suman a la totalidad. Al igual que los análisis matemáticos, los sistemas complejos se encuentran limitados al tratar de derivar consecuencias dinámicas a partir de un múltiple número de variables, volviéndose muy dificultoso intentar imaginar todas las posibilidades que un sistema real podría exhibir, o bien predecir los efectos que éstas podrían generar. Si resulta difícil cuantificar cualquier sistema en términos de unas pocas variables, en los sistemas no-lineales o complejos es aún más dificultoso poder medir las condiciones iniciales o realizar predicciones de mediano o largo plazo. La teoría de sistemas complejos asume que las relaciones entre las distintas partes del sistema no resultan bien conocidas, son inestables, no lineales y por lo tanto no predecibles. De este modo, se vuelve muy dificultoso poder estudiarlos de forma analítica, puesto que no existen ecuaciones 


\section{perifèria}

Número 18(2), diciembre 2013

http://revistes.uab.cat/periferia

que permitan describir el comportamiento del sistema junto a los fenómenos emergentes que estos presentan; en cambio, sí pueden ser estudiados los patrones que emergen a partir del diseño de modelos computacionales y de la ejecución y validación de las simulaciones.

La Simulación Basada en Agentes es utilizada para ejecutar experimentos teóricos que permitan explorar los mecanismos que subyacen a los patrones observados, intentando enriquecer la comprensión de los procesos fundamentales que pueden emerger a partir de una variedad de aplicaciones computacionales (Axelrod, 1997a). En este enfoque convergen la simulación computacional, el modelado basado en agentes- MBA (una técnica de la inteligencia artificial) y las ciencias sociales, cuyo fin es modelizar sistemas formados por agentes autónomos que interactúan unos con otros (Epstein y Axtell, 1996; Gilbert y Abbott, 2005; Epstein, 2006; Miller y Page, 2007). Técnicamente, el objetivo de MBA es crear programas que interactúen inteligentemente con su entorno, en función del diseño de programas de agentes heterogéneos, con parámetros que definen sus habilidades 0 potencialidades y con objetivos claros de comportamiento. De este modo se concibe que los agentes puedan interactuar intercambiando información y utilizando protocolos de comunicación, aprendiendo, adaptándose, reaccionando frente al ambiente y cambiando las reglas de comportamiento. Básicamente, un Sistema Basado en Agentes se compone de tres elementos principales: agentes, entorno y reglas (Epstein y Axtell, 1996).

El propósito de utilizar MBA en arqueología es simular el comportamiento de seres humanos que viven en un ambiente virtual, definido sobre la base de teorías sociales y datos históricos. Se ha propuesto a MBA como un laboratorio de las ciencias sociales, porque permite la manipulación de variables, la observación de procesos espaciales y temporales en minutos, correlacionar los datos que proyectan los cálculos computacionales, establecer condiciones iniciales y experimentar con los posibles comportamientos emergentes, probar hipótesis, replicar los experimentos y correlacionar los resultados con los datos empíricos. En la modelización los supuestos no se esconden, deben explicitarse, permitiendo testar la consistencia interna del modelo, su consecuencia lógica o implicaciones y las 


\section{perifèria}

Número 18(2), diciembre 2013

http://revistes.uab.cat/periferia

relaciones que mantienen con los datos. Por consiguiente permite experimentar virtualmente con comportamientos que de otra manera no podríamos observar, ni replicar. Una vez puesto en marcha el programa no sólo se puede seguir la evolución de una posible configuración social, sino que también puede modificarse y reiniciarse cambiando las condiciones iniciales y permitiendo la observación de diferentes proyecciones de una dinámica social.

\section{Un Modelo de Simulación de cazadores-recolectores Patagónicos}

El modelo de simulación desarrollado para analizar a los grupos cazadores recolectores patagónicos fue diseñado con el fin de observar la dinámica de interacción entre diferentes grupos étnicos, la dinámica de fisión y fusión entre las bandas de cazadores recolectores, para evaluar qué tipo de configuraciones tienen mayor probabilidad de ocurrir. Nuestro modelo de sociedad artificial cuenta con tres elementos y un conjunto de atributos introducido en base a inferencias probabilísticas:

-Agentes (sexo, edad, lenguaje, tiempo de vida, capacidad de reproducción y producción)

-Ambiente (diferencia en la capacidad de carga)

- Reglas (parentesco y reciprocidad)

Una articulación teórica metodológica desde una propuesta etnoarqueológica permite a nivel inicial utilizar datos de estudios etnográficos e históricos de los grupos históricos de Patagonia continental (Mapuche, Günün-kunne, Tehuelche, etc.) y diferentes teorías de formación cazadora-recolectora, conformando el cuerpo básico sobre el cual se derivarán las simulaciones de las poblaciones humanas. De este modo, se espera que los resultados nos permitan probar un modelo de desarrollo que reconozca los recursos, las transformaciones y las permanencias de las fronteras étnicas y culturales y así nos habilite a comprender la configuración de la diversidad socio-cultural patagónica.

De acuerdo a los datos etnológicos, arqueológicos e históricos, durante la mayor parte de su historia las poblaciones humanas patagónicas construyeron sistemas de 


\section{perifèria}

Número 18(2), diciembre 2013

http://revistes.uab.cat/periferia

producción cazadores recolectores con suficiente flexibilidad para ser capaces de explotar diferentes recursos en diferentes lugares y con diferentes intensidades. En este caso, las decisiones sociales y políticas estuvieron limitadas (condicionadas, ya que no determinadas) por la disponibilidad espacial de algunos recursos, por la ausencia de otros y por la no predictibilidad de la productividad natural.

El modelo simulado, por tanto, no es una hipótesis a comprobar, sino una plataforma de producción de hipótesis verosímiles en distintos escenarios imaginables y Patagonia constituye uno de esos escenarios.

La plataforma de simulación utilizada es Netlogo (Uri Wilensky 1999), la cual permite trabajar en un mundo bidimensional compuesto por tres tipos de agentes: patches, tortugas y observador. El comportamiento global es representado como un conjunto de entidades cuyas acciones son realizadas en función de sus características, al mismo tiempo permite introducir variabilidad a partir de la definición heterogénea de los agentes en base a diferentes propiedades que pueden ser modificadas temporalmente. Las consecuencias dinámicas o propiedades que emergen a partir de los supuestos teóricos es una diferencia básica con respecto a los modelos de dinámica linear cuyo comportamiento sólo es expresable a través de la suma de comportamientos de sus descriptores.

\section{Incidencia de la movilidad de los agentes sobre la conformación de grupos}

En cuanto a la movilidad de los agentes en el modelo, uno de los parámetros experimentados ha sido el Radio movilidad de los agentes, que permite establecer la distancia máxima de movilidad o desplazamiento de los agentes, representando la capacidad de movilidad para cada ciclo anual. Dado que todos los agentes están limitados por el mismo parámetro, la probabilidad de interactuar con un agente compatible con los cuales interaccionar favorece los patrones de movilidad entre los agentes, evitando de este modo el mantenimiento de divisiones grupales cerradas. Hemos experimentado cómo incide el Radio de movilidad en la conformación de los grupos étnicos, simulando cómo su variación afecta las probabilidades de 


\section{perifèria}

Número 18(2), diciembre 2013

http://revistes.uab.cat/periferia

interacción entre las unidades familiares.

Los resultados reflejaron que al aumentar el radio de vecindad, se registra un aumento en la fusión de los grupos y del mestizaje cultural, desapareciendo la mayoría de los grupos segregados que progresivamente van integrándose en una agregación mayor. Como señaló Cavalli-Sforza, para comprender la diferenciación cultural es necesario entenderla como un proceso determinado por la transmisión cultural en posición horizontal y por los mecanismos de dispersión de la población. Se asume que la movilidad de los agentes debe fomentar la convergencia de la globalización cultural en la medida en que actúa como una especie de acoplamiento global entre ellos. La movilidad de los agentes es impulsada por la subsistencia y por las interacciones con otros agentes de su Vecindario: cuanto mayor es el territorio que efectivamente se recorre en una unidad de tiempo dada, mayor la probabilidad de encontrarse con alguien culturalmente "semejante". En este experimento hemos podido comprobar cómo el tamaño del territorio recorrido por cada agente durante un ciclo de la simulación también afecta el grado de etnogénesis. Así, cuando el radio de movilidad aumenta, aumenta también la probabilidad de interacción social entre agentes espacialmente distantes. De este modo la "cultura" se difunde a través de un proceso de imitación local, con un grado de probabilidad fijo, cada agente puede copiar alguno de los rasgos culturales de entre los más frecuentes (moda estadística) en el grupo local al que pertenece, aumentando así el consenso y la homogenización cultural. Recordemos que estos rasgos también mutan afectados por una tasa de cambio aleatoria, con valores bajos en los inicios de la simulación pero que van incrementando pudiendo alterar a largo plazo los niveles iniciales de semejanza. Los cambios aleatorios producidos en el vector "cultural" se van acumulando, lo que provoca el aumento de la diferencia y la disminución de la frecuencia de interacción.

Nuestra simulación ha conseguido identificar en qué grado actúan los mecanismos de agregación y segregación en sociedades de pequeña escala. Como han reflejado los resultados obtenidos de las diversas pruebas de simulación efectuadas a partir del modelo de simulación Patagónico, se ha demostrado que la emergencia de grupos culturalmente diferenciados no depende directamente del tamaño de la 


\section{perifèria}

Número 18(2), diciembre 2013

http://revistes.uab.cat/periferia

Población: los grupos pueden emerger incluso cuando el tamaño de la población se mantiene constante y no se registren densidades demográficas superiores a la cantidad de recursos explotables en el área geográfica dada. Podría decirse que la simulación confirma que el proceso de etnogénesis es un proceso socialmente mediado alimentado principalmente por relaciones sociales, que caracterizan la relación dialéctica existente entre los mayores rendimientos de la cooperación económica (caza colectiva), el nivel de tolerancia cultural y los umbrales de subsistencia.

La simulación también ha mostrado cómo los procesos concretos de interacción entre grupos e individuos no tienen por qué estar determinados por el ambiente físico. Por simple que sea el mecanismo de obtención de la subsistencia, las decisiones que hacen referencia a la interacción (agregación/segregación, fusión/fisión) están motivadas por decisiones políticas, racionales en el corto plazo, impredecibles en el largo. Y la expresión de esa posible "irracionalidad" aparece en la movilidad física de las personas, tanto en el caso histórico como en el simulado: los agentes se mueven "de la misma manera" ya sea por condicionante ambiental, ya sea por determinación política.

A modo de cierre, proponemos MBA como una herramienta exploratoria, en la que no contrasta la teoría con la observación, pero si sugiere los mecanismos que pueden generar desconcierto en los patrones empíricos. Bajo la ausencia de evidencia arqueológica los modelos analíticos intentan reconstruir el comportamiento de los grupos cazadores recolectores patagónicos utilizando los fenómenos observables como modelo de referencia y buscando explicar los patrones observados del comportamiento del sistema social. 


\section{perifèria}

Número 18(2), diciembre 2013

http://revistes.uab.cat/periferia

\section{Bibliografía}

Axelrod, Robert (1997). "Advancing the Art of Simulation in the Social Sciences" en R. Conte et.al. (Eds.) Simulating Social Phenomena, Lecture Notes in Economics and Mathematical Systems 456. Berlin: Springer, pp. 21-40.

Barcelo, Joan A.; CUESTA, José; Del Castillo, Florencia; Galan, José M.; Mameli, Laura ; Miguel, Francesc; Santos, José J. y Xavier Vila (2010). "Simulating Social, Economic and Political Decisions in a hunter-Gatherer group. The case of prehistoric Patagonia". Computer Applications in Archaeology Conference CAA2010, Granada.

Cavalli-Sforza, Luigi (1997). "Genes, Peoples and Languages". Proc. National Academy of Sciences, 94: 7719-7724. USA.

Epstein, Joshua (2006). Generative Social Science. Studies in Agent-Based Computational Modeling. Princeton: Princeton University Press.

Epstein, Joshua y Robert Axtell. (1996). Growing Artificial Societies: Social Science from the Bottom up. Cambridge, MA: Brookings Institution Press MIT.

Gilbert, Nigel y Andrew Abbott. (2005). "Introduction". American Journal of Sociology, vol. $110, \quad 4$, pp. 859-863.

Miller, John H. y Scott E. Page. (2007). Complex Adaptive System. An Introduction to Computational Models of Social Life. Prince-ton: Princeton. University Press.

Wilensky, Uri (1999). Center for Connected Learning and Computer-Based Modeling, Northwestern University. Evanston, IL. Available from: http://ccl.northwestern.edu/netlogo/ 